\title{
Influence of waveguide dispersion on short-pulse free electron laser detuning curves
}

\author{
Vitali Zhaunerchyk, ${ }^{1, *}$ Dick Oepts, ${ }^{2}$ Rienk T. Jongma, ${ }^{1}$ and Wim J. van der Zande ${ }^{1}$ \\ ${ }^{1}$ Radboud University Nijmegen, Institute for Molecules and Materials, Heyendaalseweg 135, NL-6525 AJ, Nijmegen, The Netherlands \\ ${ }^{2}$ FOM Institute for Plasma Physics "Rijnhuizen", P.O. Box 1207, 3430 BE Nieuwegein, The Netherlands
}

(Received 20 September 2011; published 1 May 2012)

\begin{abstract}
In this paper we present results of numerical studies on the cavity desynchronization in the short-pulse waveguided free electron laser (FEL) oscillator FLARE, which is a new THz FEL at the Radboud University Nijmegen, with the emphasis to investigate the influence of the waveguide dispersion. In particular, the peaks of the detuning curves are predicted to be essentially broader. We also predict that the extent of the peak broadening increases as the waveguide dispersion becomes more significant.
\end{abstract}

DOI: 10.1103/PhysRevSTAB.15.050701

PACS numbers: 41.60.Cr

\section{INTRODUCTION}

Free electron lasers (FELs) are powerful versatile light sources, in which the light is emitted by relativistic electrons propagating through a periodic magnetic field created by an undulator. The main advantage of FELs over conventional lasers is that they do not have restrictions on the generated frequencies. Any frequency can be selected by a proper choice of undulator parameters and electron energy. Tunable FELs have been realized at both extremes of the spectral range, at $\mathrm{x}$-ray and $\mathrm{THz}$ frequencies $[1,2]$, regions of the spectrum inaccessible for conventional lasers.

Most FELs are driven by radio-frequency linear accelerators (rf linacs) producing intense few ps short electron bunches. The first FELs driven by rf linacs were constructed to operate at small slippage parameters (see, e.g., Ref. [3]). The slippage parameter is defined as

$$
\mu_{\text {slip }}=\frac{N_{u} \lambda_{r}}{\sigma}
$$

where $N_{u}$ is the number of undulator periods, $\lambda_{r}$ is the FEL light resonant wavelength, and $\sigma$ is the length of the electron bunch. Since electrons propagate slower than the speed of light $c$, at the exit of the undulator the front of the optical pulse will be ahead of the electron bunch by the distance $N_{u} \lambda_{r}$, the slippage distance. At large slippage parameters the electrons interact only with a part of the optical pulse and short-pulse effects become important. One such effect in the FEL oscillators is "lethargy" $[4,5]$. This effect implies that in the perfectly synchronized cavity, i.e., when the optical pulse round-trip time is equal

\footnotetext{
*Current address: Department of Physics, Albanova University Centre, Stockholm University, SE 106 91, Stockholm, Sweden. zhaunerchyk@science.ru.nl

Published by the American Physical Society under the terms of the Creative Commons Attribution 3.0 License. Further distribution of this work must maintain attribution to the author $(s)$ and the published article's title, journal citation, and DOI.
}

to the period of electron bunches, electrons preferentially amplify only the rear part of the pulse, which results in a significant decrease of the overall single-pass gain and hampers the buildup of the optical energy. The "lethargy effect" can be compensated via the cavity desynchronization meaning a reduction of the cavity length (see, e.g., Ref. [6]).

For a long wavelength FEL a waveguide is usually employed in order to suppress diffraction losses. The waveguide dispersion links the wave number $k_{z}$ and the light frequency $\omega$ as follows:

$$
k_{z}=\frac{1}{c} \sqrt{\omega^{2}-\omega_{c}^{2}}
$$

where $\omega_{c}$ is the cutoff frequency, which corresponds to the lowest frequency of the light that can propagate through the waveguide. Accordingly, the optical group velocity depends on $\omega$ as

$$
v_{g}=\frac{\partial \omega}{\partial k_{z}}=c^{2} \frac{k_{z}}{\omega} .
$$

Since in the waveguided FEL the optical pulses move with $v_{g}$ smaller than $c$, the slippage distance will be smaller than in open resonator FELs. The slippage distance in this case is given by $N_{u} \lambda_{r} \Delta$, where $\Delta$ is the FEL waveguide parameter [7]. $\Delta$ corresponds to 0 when the group velocity of the light is the same as the electron velocity and to 1 when the group velocity equals $c$ as in an open resonator FEL. Because of the waveguide dispersion, two resonant frequencies are supported in a waveguided FEL. In one case the optical pulse propagates faster than the electrons (higher frequency branch), in the other case the situation is reverse (lower frequency branch) [7]. It has recently been reported that selective amplification of the lower frequency branch is feasible for FELs employing electron bunches shorter than the corresponding light wavelength [8]. However, in most cases amplification of the higher frequency branch is preferred and in this paper we concentrate only on this branch. In particular, for this branch 
we numerically investigate cavity desynchronization in a short-pulse waveguided FEL. Whereas this is well studied for the open resonator FELs [6,9-17], in this paper we describe how detuning curves are altered by the waveguide dispersion. The results reported here have been obtained for the free electron laser for advanced spectroscopy and high-resolution experiments (FLARE), which is a new THz FEL oscillator under construction at the Radboud University Nijmegen. FLARE is distinct among other short-pulse FELs in that it will operate at very large slippage parameters, which can be as large as 40 . In the paper we first briefly describe FLARE, then the numerical model utilized to investigate the FLARE dynamics, and after that the simulation results are presented.

\section{FLARE DESCRIPTION}

The layout of FLARE is shown in Fig. 1. The rf linac accelerates electrons to $10-15 \mathrm{MeV}$ energies and produces $10 \mu$ s long electron macrobunches each $0.1 \mathrm{~s}$. The macrobunches consist of short $200 \mathrm{pC}$ microbunches with a duration of $\approx 3$ ps generated at a rate of $\approx$ $3 \mathrm{GHz}$. This repetition rate implies that 150 optical pulses will propagate simultaneously through the $7.5 \mathrm{~m}$ long FLARE optical cavity. The undulator is planar and has 40 periods with $0.11 \mathrm{~m}$ length. The waveguide employed at FLARE consists of two parallel plates separated by $10 \mathrm{~mm}$, which are placed perpendicular to the undulator magnetic field. Because of this, in the wiggle plane the light propagates as a Gaussian beam. The curvature radius of the cylindrical aluminum mirrors, $R=4.81 \mathrm{~m}$, defines the Rayleigh distance to be $2 \mathrm{~m}$. The upstream mirror is designed to be movable in order to enable cavity length detuning. The optical energy stored in the cavity is outcoupled via a rectangular slit with a variable width mounted in the downstream mirror perpendicular to the waveguide plates. The full length between the cavity mirrors is waveguided. The undulator magnetic field can be set from 0.1 to $0.47 \mathrm{~T}$ by tuning the gap between the magnets. The FLARE parameters are summarized in Table I.

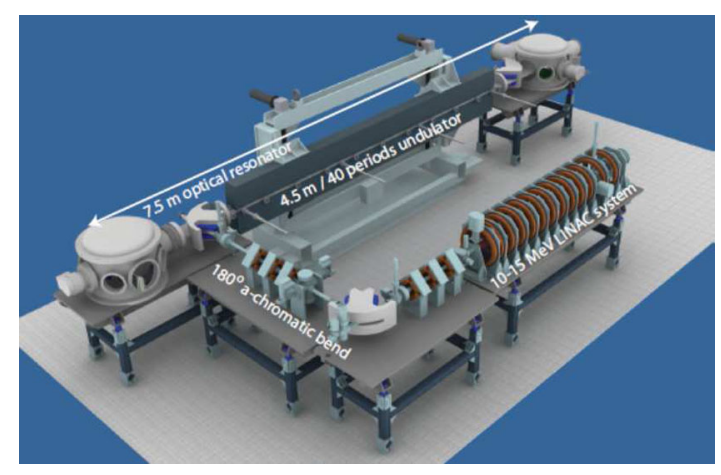

FIG. 1. 3D artist impression of FLARE.
TABLE I. FLARE parameters.

\begin{tabular}{lc}
\hline \hline Optical wavelength range & $100-1600 \mu \mathrm{m}$ \\
\hline rf frequency & $2.997924 \mathrm{GHz}$ \\
Electron beam energy & $10-15 \mathrm{MeV}$ \\
Electron bunch duration & $3 \mathrm{ps}$ (standard deviation) \\
Electron bunch charge & $200 \mathrm{pC}$ \\
Undulator period & $110 \mathrm{~mm}$ \\
Number of undulator periods & 40 \\
Undulator magnetic field & $0.1-0.47 \mathrm{~T}$ \\
Optical cavity length & $7.5 \mathrm{~m}$ \\
Total round-trip power losses & $22 \%$ \\
Outcoupled power & $7 \%$ \\
Waveguide gap & $10 \mathrm{~mm}$ \\
Mirrors curvature radius & $4.81 \mathrm{~m}$ \\
Rayleigh distance & $2 \mathrm{~m}$ \\
\hline \hline
\end{tabular}

\section{NUMERICAL MODEL}

Most of the existing FEL models employ averaging over a ponderomotive potential period or resonant wavelength. However, such models are not valid for the lower frequency part of the FLARE spectral range, where the optical wavelengths are comparable to the longitudinal size of the electron bunches (Table I). Therefore, a model that considers motion of individual electrons must be utilized to correctly describe the FLARE dynamics. Since the rf linac produces electron bunches with more than $10^{9}$ electrons, consideration of individual electrons would computationally be very expensive and a macroparticle approach is employed instead [18-21]. In this approach, the electron bunch is decomposed into pieces, containing a certain amount of electrons, and each piece is regarded in the simulation as an independent structureless macroparticle with a constant charge. Space-charge effects, i.e., mutual interaction between electrons, are assumed to be negligible and, therefore, are excluded from the simulation. In the simulation we also assumed the initial kinetic energies of electrons to have no spread and a zero transverse emittance of the electron bunch. Motion of the macroparticle in the electric and magnetic fields is described by the Lorentz force equation:

$$
\frac{d \mathbf{v}_{i}}{d z}=\frac{1}{\gamma_{i}}\left\{-\frac{e}{m_{e}} \frac{1}{v_{z i}}\left(\mathbf{E}\left(\mathbf{r}_{i}, t_{i}\right)+\left[\mathbf{v}_{i} \times \mathbf{B}\left(\mathbf{r}_{i}, t_{i}\right)\right]\right)-\mathbf{v}_{i} \frac{d \gamma_{i}}{d z}\right\}
$$

and the Lorentz factor $\gamma_{i}$ is found from [20]

$$
\frac{d \gamma_{i}}{d z}=-\frac{e}{m_{e} c^{2}} \mathbf{v}_{i} \cdot \mathbf{E}\left(\mathbf{r}_{i}, t_{i}\right)
$$

where $i$ refers to a certain macroparticle, $z$ is the direction of propagation, $\mathbf{v}$ is the macroparticle velocity, $m_{e}$ and $e$ are the electron mass and charge, respectively, $\mathbf{E}(\mathbf{r}, t)$ is the optical electric field, and $\mathbf{B}(\mathbf{r}, t)$ is the overall magnetic field of the optical pulse and undulator. 
In the model the electric and magnetic fields are presented in the frequency domain and are expanded via a complete set of orthogonal modes. For instance, the electric field is presented as

$$
\mathbf{E}(\mathbf{r}, \omega)=\sum_{m, n} C_{m, n}(z, \omega) \Psi_{m, n},
$$

where $\left\{\Psi_{m, n}\right\}$ is the complete set of the Hermite-Gaussian modes $[22,23]$ and $C_{m, n}$ is the slowly varying amplitude of the particular mode. $\mathbf{E}(\mathbf{r}, \omega)$ relates to $\mathbf{E}(\mathbf{r}, t)$ via the Fourier transform. The amplitude coefficients are found from the following excitation equation [24]:

$$
\frac{d C_{m, n}(z, \omega)}{d z}=-\frac{1}{4 P_{m, n}} \int \mathbf{J}(\mathbf{r}, \omega) \Psi_{m, n}^{*} d A
$$

where $\mathbf{J}(\mathbf{r}, \omega)$ refers to the electron current density and $P_{m, n}$ is the normalization power of the particular mode. The dynamical equations for each macroparticle [Eqs. (4) and (5)] were solved self-consistently with the excitation equations [Eq. (7)] employing the Cash-Karp method [25]. It is assumed that only the Hermite-Gaussian modes with $n=1$ and $m=0$ are excited in the cavity [22,23]. The intracavity and outcoupled losses were assumed to be independent of the wavelength. In order to reduce computational time, instead of evaluating 150 optical pulses, all the pulses were supposed to be identical and only a single pulse was considered in the simulation. The computational time was further reduced by means of parallelization of the code [26].

\section{NUMERICAL RESULTS AND CONCLUSIONS}

The resonant wavelengths and the corresponding FLARE parameters for which calculations have been performed are listed in Tables I and II. Figures 2(a) and 2(b) illustrate the detuning curves, i.e., outcoupled saturated optical energy at different cavity detuning values, $\delta L$, obtained at these resonant wavelengths. Note, desynchronization means reduction of the cavity length, hence $\delta L$ is negative.

The shape of the detuning curves calculated numerically at the short resonant wavelengths, $100 \mu \mathrm{m}$ and $256 \mu \mathrm{m}$ [Fig. 2(a)], are similar to those of the open resonator FELs, which also show a sharp peak (e.g., see Ref. [11]). The $\Delta$ parameters are close to unity for both $\lambda_{r}=100 \mu \mathrm{m}$ and $\lambda_{r}=256 \mu \mathrm{m}$ (Table II), which implies that at the

TABLE II. Resonant wavelengths and the corresponding FLARE parameters.

\begin{tabular}{lcccccc}
\hline \hline$\lambda_{r}, \mu \mathrm{m}$ & 100 & 256 & 748 & 990 & 1230 & 1592 \\
\hline$E_{b}, \mathrm{MeV}$ & 15.0 & 13.0 & 11.9 & 11.9 & 11.9 & 11.0 \\
$B_{u}, \mathrm{~T}$ & 0.11 & 0.2 & 0.34 & 0.39 & 0.43 & 0.44 \\
$\Delta$ & 0.97 & 0.93 & 0.79 & 0.73 & 0.66 & 0.56 \\
\hline \hline
\end{tabular}

short wavelengths FLARE resembles an open resonator short-pulse FEL. In such FELs the synchronism between optical pulses and the electron bunches cannot be sustained simultaneously during optical energy growth and during saturation. Only close to the zero detuning the electron bunches and optical pulses are synchronized at saturation resulting in the sharp peak in the detuning curves. However, since the electronic gain is very small [Fig. 2(a), the dashed curve], the buildup of optical energy is slow (the "lethargy effect"). In contrast, at the highest gain, which occurs with the shorter cavity, the round-trip time of circulating optical pulses is reduced such that during the buildup of the intracavity energy it is synchronized to the repetition rate of the rf linac. In this case, the optical energy is saturated much faster, but it stays at a lower level. Further shortening of the cavity reduces the optical pulse round-trip time, thereby disrupting the synchronism with electron bunches, which diminishes the FEL gain [Fig. 2(a), the dashed curve]. Figure 2(c) shows the FEL efficiency at saturation for $\lambda_{r}=100 \mu \mathrm{m}$ and $\lambda_{r}=$ $256 \mu \mathrm{m}$ versus different relative detuning values, $\delta L / \lambda_{r}$, and the dashed curves in the figure depict FWHM bandwidth of the optical pulses, $\Delta \omega / \omega$, calculated at $\lambda_{r}=$ $100 \mu \mathrm{m}$ in the small-signal regime. From the figure, it follows that the duration of the optical pulses increases as the cavity length is shortened. Reduction of the cavity length suppresses the "lethargy effect," which improves the overlap with electrons and stretches the optical pulses. As was suggested in Ref. [9], the FEL efficiency increases with the optical pulse bandwidth and, indeed, such a relation is observed in Fig. 2(c).

Waveguide dispersion has effects on the detuning curves, which become more pronounced at long wavelengths. Figure 2(b) shows that at the long wavelengths the optical pulses propagate through the waveguide with $v_{g}$ smaller than $c$ [Eq. (3)] and the FEL cavity has to be shortened in order to preserve synchronization between the electron bunches and the light pulses and to achieve lasing. While the wavelength increases, the total range of the detuning values that allows lasing decreases relative to $\lambda_{r}$ and this effect is stressed in Fig. 2(d), which shows the efficiency (saturation power) against $\delta L / \lambda_{r}$. Such a tendency is a consequence of the relative slippage distance, $N_{u} \Delta$, that reduces with increasing wavelength (Table II), i.e., with smaller $N_{u} \Delta$ the shorter optical pulses relative to $\lambda_{r}$ are produced and, respectively, the range of the $\delta L / \lambda_{r}$ values is reduced. The fact that the maximum efficiency decreases with increasing wavelength [Fig. 2(d)] is due to the large diffraction at long wavelengths, which worsens the overlap between the light field and the electrons.

The detuning curves shown in Fig. 2(b) have remarkably broader main peaks compared to those at the shorter wavelengths [Fig. 2(a)]. In Figs. 2(c) and 2(d), the curves are shown on a scale normalized to the resonant wavelength, 

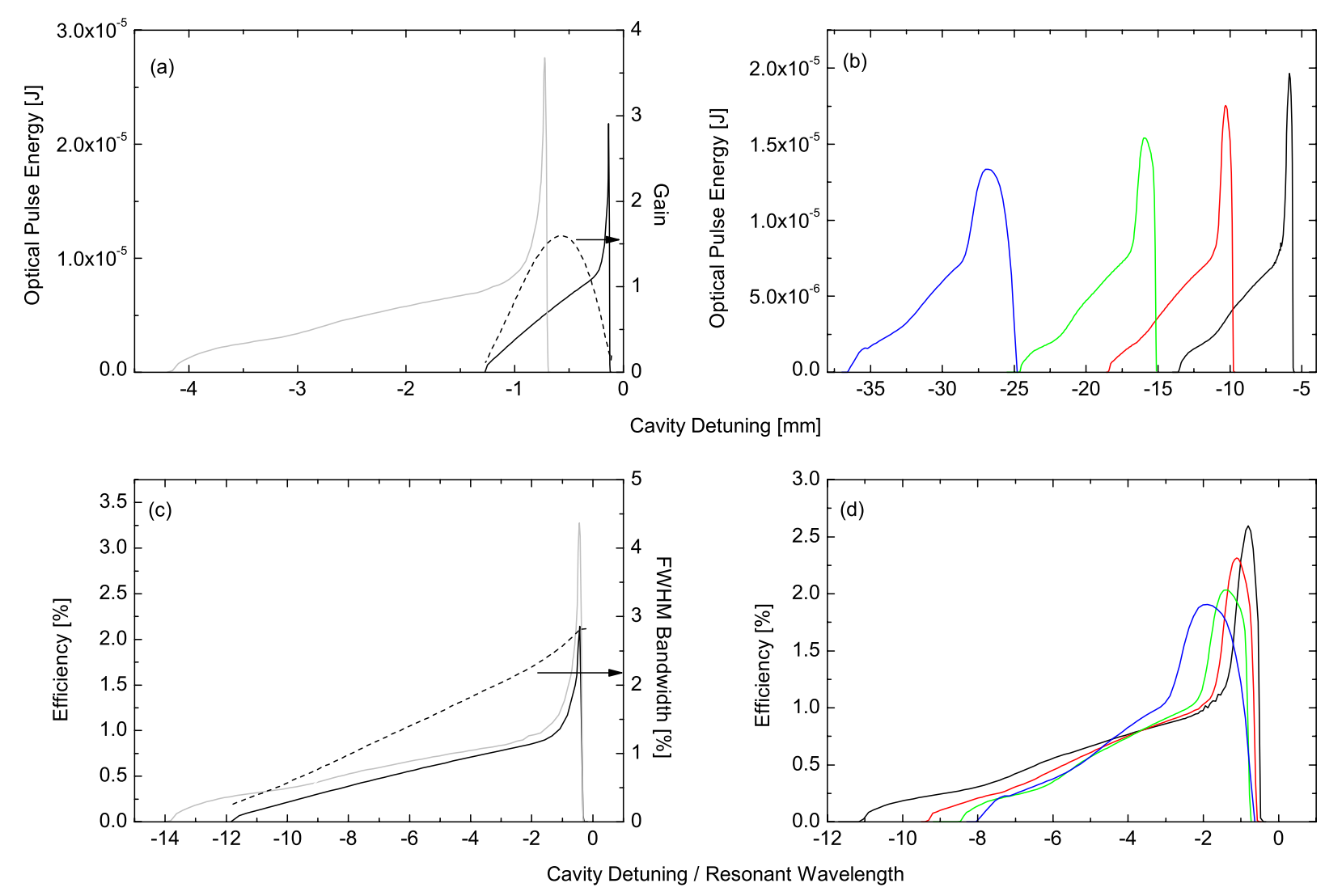

FIG. 2. The left and right sides of the figure correspond to the results obtained at the short resonant wavelengths of $100 \mu \mathrm{m}$ (black curve) and $256 \mu \mathrm{m}$ (gray curve), and for the long resonant wavelengths of $748 \mu \mathrm{m}$ (black curve), $990 \mu \mathrm{m}$ (red curve), $1230 \mu \mathrm{m}$ (green curve), and $1592 \mu \mathrm{m}$ (blue curve), respectively. (a),(b) Detuning curves obtained after averaging over 50 oscillator passes at the end of the macrobunch. (c),(d) The FEL efficiency at saturation versus different detuning values and for each curve $\delta L$ values are divided by the corresponding resonant wavelength. $\delta L=0$ corresponds to the cavity length of $v_{g} t_{b} / 2$, where $v_{g}$ is the group velocity found according to Eq. (3) for a given $\lambda_{r}$ and $t_{b}$ is the period between successive electron bunches multiplied by 150 . The dashed curves in (a) and (c) depict the electronic gain and FWHM optical pulse bandwidth, respectively, calculated at $\lambda_{r}=100 \mu \mathrm{m}$ in the small-signal regime. The scales for the gain and bandwidth are shown on the right side abscissa in (a) and (c), respectively.

which clearly demonstrates that the width of the main peaks increases with $\lambda_{r}$. Furthermore, the highest efficiency tends to occur at the larger $|\delta L| / \lambda_{r}$ values [Fig. 2(d)]. In order to understand the origin of the peak broadening, we analyze the saturated optical spectral density versus different cavity lengths calculated at $\lambda_{r}=$ $1592 \mu \mathrm{m}$ (Fig. 3), for which the strongest broadening is predicted. When the stored optical energy increases, the electrons lose a greater amount of their energy in the undulator. As a result, the wavelength of the emitted light slightly increases. At some point the FEL resonant conditions are not fulfilled any longer and the stored optical energy is saturated. Such a shift from $\lambda_{r}$ towards longer wavelengths is obviously seen in Fig. 3 at $\delta L$ around $-27 \mathrm{~mm}$. However, at the larger $|\delta L|$ values the FEL efficiency, as is illustrated in Fig. 2(d), continuously diminishes and the increase in emitted wavelength becomes less pronounced (Fig. 3). Furthermore, due to the waveguide dispersion, the propagation velocity depends on the

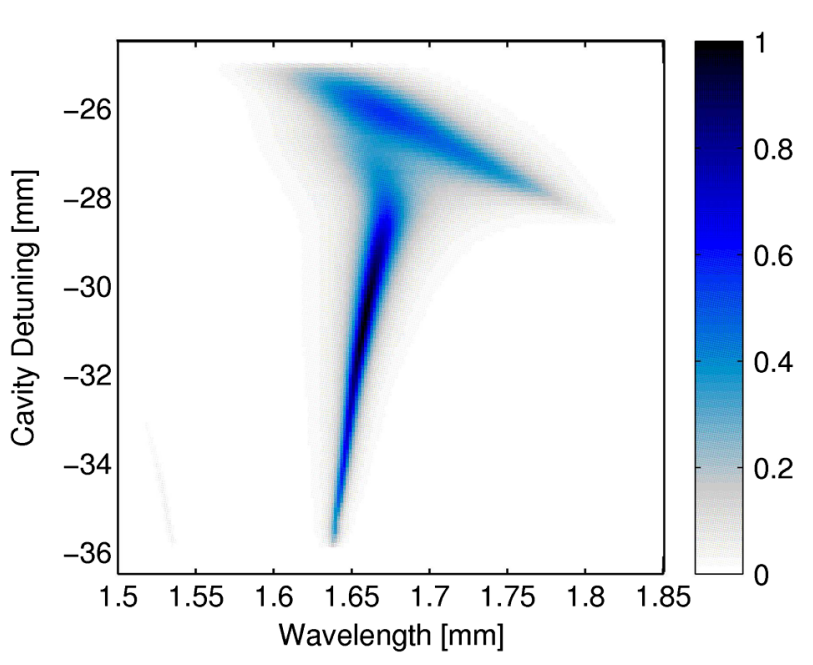

FIG. 3. Relative optical spectral density obtained at saturation for the resonant wavelength of $1592 \mu \mathrm{m}$ versus different cavity detuning values. 

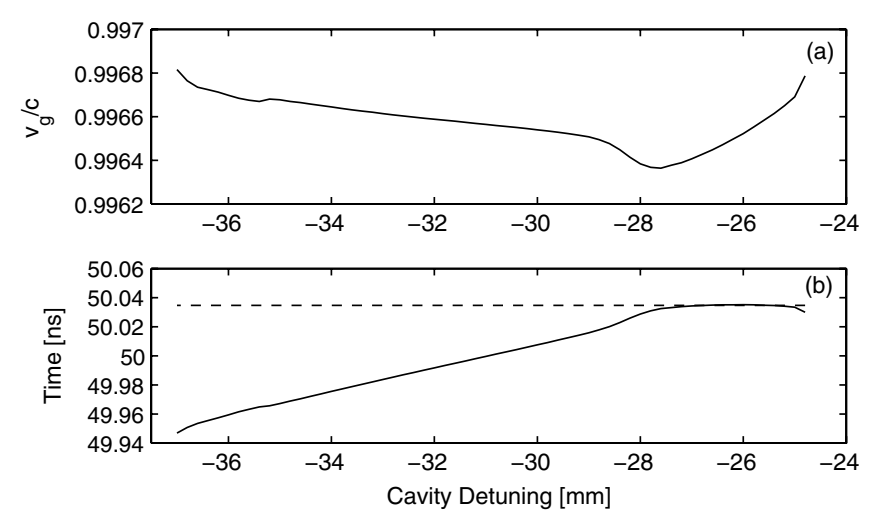

FIG. 4. The resonant wavelength is $1592 \mu \mathrm{m}$. The group velocities and the corresponding round-trip times of the optical pulses calculated at the saturation are presented in (a) and (b), respectively. The dashed line in (b) is the period between successive electron bunches multiplied by 150 .

wavelength. Hence, a shift of the emitted wavelength through the waveguide affects the synchronism of optical pulses with electron bunches. Figure 4(a) shows the group velocity at different $\delta L$ values calculated according to Eq. (3) with the $\omega$ value being the mean frequency of the optical spectral density at saturation (Fig. 3). Simultaneous reduction in the group velocity with the cavity length in the region of $-28 \mathrm{~mm}<\delta L<-25.5 \mathrm{~mm}$ [Fig. 4(a)] leads to approximately constant optical pulse round-trip time, $2(L-|\delta L|) / v_{g}$, which is nearly equal to the period of the electron bunches as they are injected into the undulator [Fig. 4(b)]. Since at the saturation the synchronism between the optical pulses and the electron bunches is nearly sustained over a broad range of the $\delta L$ values [Fig. 4(b)], the corresponding detuning curve possesses the broad peak. The above distinguishes our waveguided FEL from an open resonator FEL. In an open resonator FEL and at the short wavelengths in FLARE, the round-trip time only depends on the cavity length, $2(L-|\delta L|) / c$. Therefore synchronization can survive only close to zero detuning, which manifests itself as the sharp peak in the detuning curve.

Finally, we can summarize the results reported in this paper. Two wavelength regions are described. For the first region $\left(\lambda_{r}=100 \mu \mathrm{m}\right.$ and $\left.\lambda_{r}=256 \mu \mathrm{m}\right)$, the waveguide dispersion is small and for the second region ( $\lambda_{r}=748 \mu \mathrm{m}, \lambda_{r}=990 \mu \mathrm{m}, \lambda_{r}=1239 \mu \mathrm{m}$, and $\lambda_{r}=$ $1592 \mu \mathrm{m})$, the waveguide dispersion influences the FEL operation. The detuning curves at the short wavelengths have sharp peaks, which are typical for open resonator short-pulse FELs [6,9-17]. At the long wavelengths, where the waveguide dispersion becomes pronounced, we conclude the following: (1) a larger cavity length reduction is required in order to achieve lasing; (2) the total range of the detuning values relative to the resonant wavelength where FLARE allows lasing decreases; (3) as predicted, the FEL efficiency diminishes due to the light diffraction; and (4) the peak of the detuning curve broadens and the maximum shifts towards larger $|\delta L|$ values.

\section{DISCUSSION}

This paper demonstrates operation of the long wavelength waveguided FEL in the short-pulse regime. The crucial issue for operation of open resonator short-pulse FELs, i.e., those FEL oscillators that operate at the slippage parameter $\mu_{\text {slip }}>1$, is the "lethargic" start-up [4,5]. The latter implies a low FEL gain which results in very slow buildup of optical energy such that tens of $\mu \mathrm{s}$, a typical duration of rf linac macrobunches, are not sufficient to achieve saturation. As was shown for open resonator short-pulse FELs [6,9-17] as well as reported here for the waveguided FEL, FLARE, operating at the resonant wavelengths of 100 and $256 \mu \mathrm{s}$, where the waveguide dispersion is not so significant, the lethargy effect can successfully be compensated via the cavity desynchronization, i.e., by shortening the cavity length. Although the FEL gain improves, the drawback of this approach is that it leads to a reduction of the saturation power and in such FELs it is not straightforward to obtain fast optical energy buildup with a large FEL efficiency at saturation. It could be accomplished, for instance, by tuning the cavity length when the intracavity energy is growing. However, it is not practically feasible at the moment to accurately tune the cavity length within such a short period of time. Otherwise, as it was reported by Bakker et al. [13], a high saturated power can simultaneously be achieved with a high gain by ramping the electron bunch repetition frequency. This paper shows that, by tuning the cavity length of FLARE when operating at the long wavelengths, we can obtain a high FEL efficiency in combination with sufficient small-signal gain. We conclude such a behavior to be an intrinsic feature of waveguided short-pulse FELs due to the waveguide velocity dispersion. As a result, this allows the production of short optical pulses of a few hundred ps duration with high peak power in the (sub)millimeter range.

\section{ACKNOWLEDGMENTS}

The authors would like to thank Kees van der Geer and Lex van der Meer from the FOM Institute for Plasma Physics "Rijnhuizen" for fruitful discussions on this subject and Leo Meerts from the Radboud University Nijmegen for assistance with performing parallel calculations. V.Z. acknowledges the Swedish Research Council for providing him with the PostDoc fellowship grant (Grant No. 623-2008-7180). FLARE is part of the NCAS project funded through the "Big Facilities" program of the Netherlands Organisation for Scientific Research (NWO). 
[1] P. Emma, K. Bane, M. Cornacchia, Z. Huang, H. Schlarb, G. Stupakov, and D. Walz, Phys. Rev. Lett. 92, 074801 (2004).

[2] G. Ramian, Nucl. Instrum. Methods Phys. Res., Sect. A 318, 225 (1992).

[3] K.E. Robinson, T. L. Churchill, D. C. Quimby, D. M. Shemwell, J. M. Slater, A. S. Valla, A. A. Vetter, J. Adamski, T. Doering, W. Gallagher, R. Kennedy, B. Robinson, D. Shoffstall, E. Tyson, A. Vetter, and A. Yeremian, Nucl. Instrum. Methods Phys. Res., Sect. A 259, 49 (1987).

[4] G. Dattoli, T. Hermsen, A. Renieri, and A. Torre, Phys. Rev. A 37, 4326 (1988).

[5] G. Dattoli, T. Hermsen, L. Mezi, A. Renieri, and A. Torre, Phys. Rev. A 37, 4334 (1988).

[6] D. A. Jaroszynski, D. Oepts, A. F. G. van der Meer, and P. W. van Amersfoort, Nucl. Instrum. Methods Phys. Res., Sect. A 296, 480 (1990).

[7] A. Doria, G. P. Gallerano, and A. Renieri, Opt. Commun. 80, 417 (1991).

[8] V. Zhaunerchyk, R. T. Jongma, Yu. Lurie, Y. Pinhasi, and W. J. van der Zande, Appl. Phys. Lett. 97, 231109 (2010).

[9] D. A. Jaroszynski, D. Oepts, J. M. Ortega, C. R. Pidgeon, and P. W. van Amersfoort, Nucl. Instrum. Methods Phys. Res., Sect. A 318, 582 (1992).

[10] D. A. Jaroszynski, R. J. Bakker, A. F. G. van der Meer, D. Oepts, and P. W. van Amersfoort, Phys. Rev. Lett. 70, 3412 (1993).

[11] D. A. Jaroszynski, R. J. Bakker, A. F. G. van der Meer, D. Oepts, and P. W. van Amersfoort, Nucl. Instrum. Methods Phys. Res., Sect. A 331, 52 (1993).

[12] R. J. Bakker, C. A. J. van der Geer, D. A. Jaroszynski, A.F.G. van der Meer, D. Oepts, and P.W. van Amersfoort, Nucl. Instrum. Methods Phys. Res., Sect. A 331, 79 (1993).
[13] R. J. Bakker, G. M. H. Knippels, A. F. G. van der Meer, D. Oepts, D. A. Jaroszynski, and P. W. van Amersfoort, Phys. Rev. E 48, R3256 (1993).

[14] R. J. Bakker, C. A. J. van der Geer, D. A. Jaroszynski, A.F.G. van der Meer, D. Oepts, and P.W. van Amersfoort, J. Appl. Phys. 74, 1501 (1993).

[15] G. M. H. Knippels, R. J. Bakker, A. F. G. van der Meer, D. A. Jaroszynski, D. Oepts, P. W. van Amersfoort, and J. N. Hovenier, Nucl. Instrum. Methods Phys. Res., Sect. A 341, ABS26 (1994).

[16] G. M. H. Knippels, R. F. X. A. M. Mols, A. F. G. van der Meer, D. Oepts, and P.W. van Amersfoort, Phys. Rev. Lett. 75, 1755 (1995).

[17] G. M.H. Knippels, A.F.G. van der Meer, R. F.X. A. M. Mols, D. Oepts, and P. W. van Amersfoort, Phys. Rev. E 53, 2778 (1996).

[18] Y. Pinhasi, V. Shterngartz, and A. Gover, Phys. Rev. E 54, 6774 (1996).

[19] Y. Pinhasi, Yu. Lurie, and A. Yahalom, Nucl. Instrum. Methods Phys. Res., Sect. A 475, 147 (2001).

[20] Y. Pinhasi, Yu. Lurie, and A. Yahalom, Phys. Rev. E 71, 036503 (2005).

[21] M. J. de Loos, C. A. J. van der Geer, S. B. van der Geer, A. F. G. van der Meer, D. Oepts, and R. Wünsch, Nucl. Instrum. Methods Phys. Res., Sect. A 507, 97 (2003).

[22] L. R. Elias and J.C. Gallardo, Appl. Phys. B 31, 229 (1983).

[23] L. R. Elias, G. Ramian, James Hu, and A. Amir, Phys. Rev. Lett. 57, 424 (1986).

[24] A. Gover, Phys. Rev. ST Accel. Beams 8, 030701 (2005).

[25] J. R. Cash and A. H. Karp, ACM Trans. Math. Softw. 16, 201 (1990).

[26] http://www.open-mpi.org/. 